\title{
X-Ray Selected EUV Galaxies: A Quest for the Faintest Extragalactic EUV Sources
}

\author{
ANTONELLA FRUSCIONE \\ Center for EUV Astrophysics, 2150 Kittredge Street, University of California, \\ Berkeley, CA $94720-5030$, USA
}

\begin{abstract}
Using data from the public archive of the Extreme Ultraviolet Explorer (EUVE) all-sky survey, we have systematically searched for extreme ultraviolet (EUV) emission (58-174 $\AA, 0.07-0.21$ $\mathrm{keV}$ ) around approximately 2500 distinct positions in the sky corresponding to known $\mathrm{X}$-ray emitting extragalactic sources. We find that $20 \mathrm{X}$-ray galaxies are EUV bright and were detected with significance above $4 \sigma$ during the $E U V E$ survey: 8 are reported here for the first time (MS 0037.7-0156, Mrk 142, M 65, EXO 1128.1+6908, M 87, Mrk 507, PKS 2005-489 and 1H 2351-315.A). 68 additional galaxies are detected with a lower significance $(3<\sigma<4)$, but the list is affected by a high percentage of spurious sources.
\end{abstract}

\section{Introduction}

Observations at the shortest $(\lambda \lesssim 175 \AA$ ) EUV wavelengths with the ROSAT Wide Field Camera (WFC) and the EUVE photometers, have established Active Galactic Nuclei (AGN) (Seyfert galaxies, BL Lacertae objects and quasars) as a distinct class of EUV source. The brightest members of this class were discovered during the WFC and the EUVE all-sky surveys (Pounds et al. 1993; Malina et al. 1994; Bowyer et al. 1994; Marshall et al. 1995, hereafter MFC; Vennes et al. 1995). Although small in number, and until very recently thought to be invisible at EUV wavelengths, galaxies detected in the EUV represent a crucial sample toward the understanding of the overall spectral energy distribution in AGN. In Seyfert galaxies, for example, two spectral components dominate the emission in the optical to X-ray range: the so called "big blue bump" (extending from the optical-UV to the EUV or soft X-ray range) and an hard X-ray power law component. Soft X-ray excesses have been detected above the hard X-ray component (e.g., Wilkes \& Elvis 1987; Walter \& Fink 1993) and they have been usually interpreted as a high-frequency tail of accretion disk spectra. However the connection between the soft X-ray excess and the optical/UV bump is still under debate (e.g., Czerny \& Życky 1994) and EUV observations may provide the missing link.

All the EUV AGN discovered to date share the common property of having been detected in X-rays. This is in part explained by the fact that the spectra of AGN are generally steep toward soft X-ray energies therefore they can compete with the catastrophic effect, at EUV wavelengths, of the absorption by the interstellar gas. Indeed, all the EUV galaxies lie in directions of low Galactic neutral hydrogen column density $\left(N(\mathrm{H} \mathrm{I}) \lessgtr 3 \times 10^{20} \mathrm{~cm}^{-2}\right)$. The brightest EUV extragalactic sources included in the published EUV catalogs were discovered by automatic source detection and analysis routines which are designed to extract the largest possible number of new sources while keeping a conservative significance level in order to avoid excessive contamination by spurious sources. In this process the high significance threshold used (e.g., $6 \sigma$ in Malina et al. 1994) eliminates many potentially interesting fainter sources. MFC analyzed the EUVE all-sky survey data immediately after the end of the survey and reported the detection of $13 \mathrm{AGN}$ at $2.5 \sigma$ or better. Additional data were collected after the first 6 months of the survey and the analysis software and calibration data have improved since the 
MFC analysis. A more in depth and systematic look at the $E U V E$ all-sky survey data to search for new and fainter EUV galaxies is therefore highly motivated.

\section{EUVE Observations and Data Analysis}

We have analyzed the entire Lex/B (58-174 $\AA$ ) data set (interstellar absorption prevents the observation of any extragalactic source longward of $\approx 100 \AA$ ) collected during the $E U V E$ all-sky survey and recently made available through the $E U V E$ public archive. The analysis was done independently from the previous all-sky survey results, taking advantage of all the improvements in the analysis software and in the calibration data made since the time of the earlier studies. We also included in our analysis "gap filling" data not previously available.

Unbiased automatic source detection and analysis routines applied to low signal-tonoise ratio data, such as the $E U V E$ sky maps, are not the best method for finding new faint extragalactic sources. This is because such methods need conservative significance thresholds to limit the number of spurious detections. MFC introduced a "biased" search to find EUV AGN by searching around a sample of about 200 "interesting" positions in the sky, i.e., where a X-ray AGN is present and its predicted EUV count rate is $>0.002$ count $\mathrm{s}^{-1}$. In this work we adopted a similar, but less restrictive, approach and did not eliminate any X-ray source a priori, but look for EUV emission at all positions in the sky corresponding to a cataloged X-ray extragalactic source (AGN and normal galaxies).

\subsection{Sample Selection}

Our sample of X-ray selected galaxies to test for EUV emission was constructed by collecting all the published lists, known to the author, of extragalactic sources classified as AGN or galaxies detected during surveys or pointed observations performed by the HEAO 1, Einstein, EXOSAT and ROSAT X-ray satellites. The total sample includes $5305 \mathrm{X}$-ray sources, 2430 of which are unique.

\subsection{Count Rate Determination}

We determined EUV position, source and background count rates, and detection significance, simultaneously at a given position in the sky, from the $E U V E$ sky maps, with the publicly accessible $E U V E$ software. The detection significance is expressed in terms of a $\chi^{2}$ value with 1 degree of freedom $\left(\approx \sigma^{2}\right.$ Gaussian) and corresponds to the probability that a detection is spurious, and it is caused by a random background fluctuation: we chose as our first detection threshold $\chi^{2}=9(\approx 3 \sigma)$. Statistically this value corresponds to a formal spurious rate probability of $2.7 \times 10^{-3}$. Out of the $2430 \mathrm{X}$-ray sources analyzed, 92 were detected with significance above $3 \sigma, 24$ of which were found to be above $4 \sigma$.

An hidden complication when searching the EUVE sky maps at low significance levels, is that spurious detections can be generated by the wing of the point spread function of bright sources. In order to eliminate this possibility with a high degree of confidence, we constructed an empirical model of the effect of the point spread function of bright sources on the surrounding pixels and compared the 92 significant detections in our sample with the First EUVE Catalog (Bowyer et al. 1994) setting conservative thresholds around all the sources in the catalog. We rejected as spurious three sources (all with $\chi^{2} \geq 16$ ) among the 92 with $\chi^{2} \geq 9$, because the source positions are possibly affected by bright white dwarfs. 


\begin{tabular}{|c|c|c|c|c|c|c|}
\hline $\begin{array}{l}\text { X-ray Source } \\
\text { Name }\end{array}$ & Other Name & Type & $\begin{array}{l}\text { Count rate } \\
\left(\text { count } s^{-1}\right)\end{array}$ & $\begin{array}{l}\text { Signif } \\
\sigma^{2}\end{array}$ & $\begin{array}{c}\log \\
N\left(\mathrm{H} \mathrm{I}^{\prime}\right)\end{array}$ & $\underset{10^{-12} \frac{\mathrm{erg}}{\mathrm{cm}^{2} \mathrm{~s}}}{\operatorname{Flux}}$ \\
\hline MS0037.7-0156 & & Sy & $0.035 \pm 0.012$ & 16.6 & 20.5 & 4.33 \\
\hline $1 \mathrm{H} 1023+513 \mathrm{C}$ & Mrk142 & Sy1 & $0.026 \pm 0.008$ & 16.6 & 20.1 & 1.36 \\
\hline 1WGA118.8+1306 & M65 & LI & $0.049 \pm 0.015$ & 23.7 & 20.3 & 3.84 \\
\hline EXO1128.1+6908 & & Sy1 & $0.022 \pm 0.006$ & 17.4 & 20.1 & 1.15 \\
\hline $1 \mathrm{H} 1226+128$ & M87 & Sy & $0.031 \pm 0.009$ & 17.8 & 20.4 & 3.06 \\
\hline $2 E 1748.8+6842$ & Mrk507 & Sy2 & $0.005 \pm 0.001$ & 18.3 & 20.6 & 0.76 \\
\hline RXJ20094-4849 & PKS2005-489 & $\mathrm{BL}$ & $0.027 \pm 0.009$ & 16.3 & 20.6 & 4.08 \\
\hline $1 \mathrm{H} 2351-315 . \mathrm{A}$ & $\mathrm{H} 2356-309$ & $\mathrm{BL}$ & $0.034 \pm 0.010$ & 19.4 & 20.1 & 1.78 \\
\hline WGA0057-2222 & TONS180 & Sy1.2 & $0.054 \pm 0.012$ & 37.6 & 20.2 & 3.42 \\
\hline $1 \mathrm{H} 0419-577$ & 1ES0425-573 & Sy & $0.036 \pm 0.006$ & 72.0 & 20.4 & 3.55 \\
\hline REJ1034+393 & $\mathrm{X} 12325$ & Sy & $0.023 \pm 0.007$ & 19.4 & 20.2 & 1.46 \\
\hline $1 \mathrm{H} 1104+382$ & Mrk421 & $\mathrm{BL}$ & $0.052 \pm 0.010$ & 50.2 & 20.2 & 3.29 \\
\hline $\mathrm{H} 1226+023$ & $3 \mathrm{C} 273$ & QSO & $0.050 \pm 0.015$ & 20.0 & 20.3 & 3.92 \\
\hline $1 \mathrm{H} 1350+696$ & Mrk279 & Sy1 & $0.024 \pm 0.005$ & 44.4 & 20.2 & 1.52 \\
\hline $1 \mathrm{H} 1415+255$ & NGC5548 & Sy1.5 & $0.029 \pm 0.008$ & 24.1 & 20.2 & 1.83 \\
\hline $1 \mathrm{H} 1430+423$ & WGAJ1428+4240 & BL & $0.029 \pm 0.007$ & 29.2 & 20.1 & 1.51 \\
\hline $1 \mathrm{H} 1429+370$ & Mrk478 & Sy1 & $0.061 \pm 0.010$ & 83.6 & 20.0 & 2.71 \\
\hline $1 \mathrm{H} 1651+398$ & Mrk501 & $B L$ & $0.038 \pm 0.007$ & 56.6 & 20.2 & 2.40 \\
\hline $1 \mathrm{H} 2156-304$ & PKS2155-304 & BL & $0.269 \pm 0.021$ & 571.7 & 20.1 & 14.1 \\
\hline $1 \mathrm{H} 2209-470$ & NGC7213 & Sy1 & $0.032 \pm 0.009$ & 20.0 & 20.4 & 3.16 \\
\hline
\end{tabular}

\subsection{Monte Carlo Random Sample Test}

On a purely statistical basis we expect 60 spurious detections at $9<\chi^{2}<16$ in our sample of 2430 sources. In order to check the reliability of the significance threshold values calculated by the public analysis software, we have performed a Monte Carlo simulation and constructed a control sample of pseudo-random positions as large as the real sample. We generated a pseudo-random sample similar to the real source distribution by converting the coordinates of the sources from celestial to galactic, inverting the sign of the galactic latitude and finally transforming the coordinates back to celestial. We analyzed the random sample the same way as the real sample: 54 random positions yielded $\chi^{2}>9,3$ of which had $\chi^{2}>16$. However all three $4 \sigma$ detections were eliminated because they coincide with EUV bright cataloged objects. In conclusion, 51 random positions (a priori not corresponding with any known or suspected EUV source) were found with significance $9<\chi^{2}<16(3<\sigma<4)$. This number is in reasonable agreement with the value of 60 obtained on purely statistical grounds. Given that $68 \mathrm{X}$-ray AGN and galaxies were found with $9<\chi^{2}<16$, only about 10 to 20 of them are likely to be real. Therefore we will concentrate from now on, on the highly reliable $4 \sigma$ sources.

\section{Discussion}

Our systematic search found that $21 \mathrm{X}$-ray selected extragalactic objects have been detected as likely sources of EUV radiation $(\sigma>4)$. To exclude any other possible candidate, we performed a catalog search in a 3 arcmin circle around the 21 positions using the NED and SIMBAD databases: in all but one case (EXO 1429.9+371 which we excluded) the $\mathrm{X}$-ray galaxy is the only cataloged object.

Table 1 gives the final list of the $20 \mathrm{X}$-ray selected AGN detected during the EUVE 


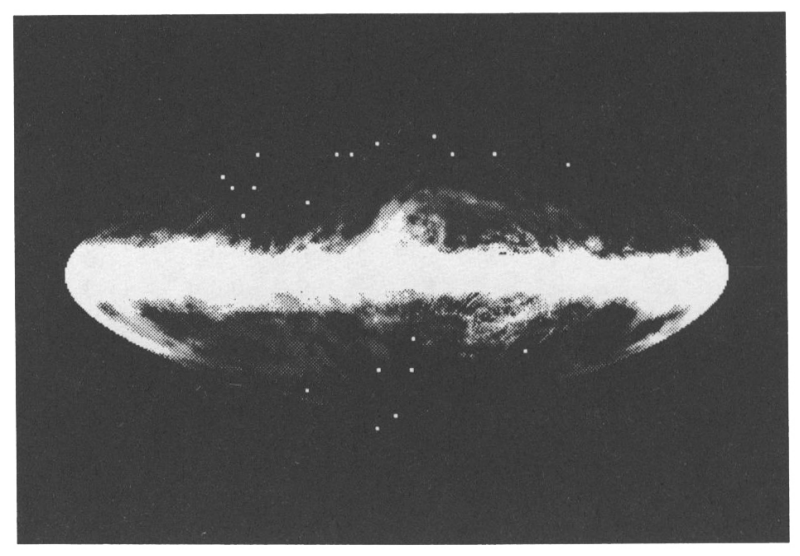

FIGURE 1. Galactic distribution of the X-ray selected AGN detected during the EUVE all-sky survey, superimposed on the $N(\mathrm{H} \mathrm{I})$ sky map (Dickey \& Lockman 1990). The map is centered on the galactic center with longitude increasing to the left.

all-sky survey. Eight of them are reported here for the first time, and are listed in the top section of the table. Twelve were previously known EUV sources (MFC, Vennes et al. 1995). Two EUV galaxies reported in MFC (NGC 4051 and $1 \mathrm{H} \mathrm{1013+489)}$ are not detected with $\chi^{2}>16$ : the first was detected at $\sigma=3.6$ in agreement with MFC results, the second showed a low significance of $2.7 \sigma$. We want to emphasize the fact that our independent analysis recovered all the previously discovered EUV AGN.

In the table we list the source type, as Sy (Seyfert), BL (BL Lac object), LI (LINER) or QSO: all the $4 \sigma$ sources are AGN. The EUV position of all the sources is within 2 arcmin of the X-ray position, and even closer to the object optical coordinates. The Lex/B count rate and the corresponding standard deviation are in column 4 . The significance (in column 5) is expressed as a $\chi^{2}$ value and it is approximately equal to the Gaussian $\sigma^{2}$. Column 6 lists the $N(\mathrm{H} \mathrm{I})$ values from Stark et al. (1992) or Dickey \& Lockman (1990) unless a more accurate narrow beam pointed $21 \mathrm{~cm}$ measurement was found in the literature (e.g., Elvis et al. 1989)-interstellar absorption (both in the Galaxy and intrinsic to the source) is the single most relevant limiting factor in the detection of EUV galaxies. As expected, the column densities in the directions of the detected EUV AGN are below $\approx 3 \times 10^{20} \mathrm{~cm}^{-2}$ in all cases but Mrk 507 and PKS 2005-489; however Mrk 507 was observed for more than $35000 \mathrm{~s}$ due to its high ecliptic latitude which could explain the detection despite the relatively high $N(\mathrm{H} \mathrm{I})$. A conspicuous demonstration of the anticorrelation between AGN detection in the EUV and $N(\mathrm{H} \mathrm{I})$ distribution is given in Figure 1 where the galactic distribution of the $20 \mathrm{X}$-ray selected EUV AGN is superimposed on the $N(\mathrm{H} \mathrm{I})$ map (Dickey \& Lockman 1990).

MFC discussed the problems related to a proper determination of the EUV flux from a single broad-band EUV count rate, namely the strong dependence on $N(\mathrm{H} \mathrm{I})$ and the fact that the EUV spectral shape is poorly known unless EUV and soft X-ray observations are taken simultaneously. We used Table 3 from MFC and the count rate from column 4 to estimate the approximate source flux reported in column 7 . We assumed the Galactic absorption in column 6 and a "sample" energy index $\alpha=2$ for all the sources (varying $\alpha$ in the $0.5-3$ interval results in a maximum of $30 \%$ change in the total flux). 
Most of the brightest EUV extragalactic sources have been studied in detail and EUV spectroscopy has been performed on 6 of them. We refer to the individual papers for comments on PKS 2155-304 (Marshall et al. 1993, Fruscione et al. 1994, Königl et al. 1995), 3C 273 (Kafatos et al. this volume), Mrk 421 (Bruhweiler et al. 1995), Mrk 478 (Marshall et al. 1995b), NGC 5548 (Kaastra et al. 1994), TON S180 (Vennes et al. 1995) and Mrk 279 (Hwang, Bowyer, \& Lampton this volume). Detailed analysis on each individual new EUV AGN will be presented in a forthcoming paper (Fruscione 1995).

\section{Conclusion}

We have systematically searched the EUVE all-sky survey data for EUV emission from a large sample of X-ray selected AGN and galaxies. 20 AGN emit detectable EUV radiation, 8 of which (6 Seyfert galaxies and 2 BL Lac objects) are reported here for the first time. This sample includes the brightest EUV extragalactic sources and it is likely to represent nearly all the known AGN that were detected during the EUVE all-sky survey. However, many unidentified sources are still listed in the EUV catalogs and could hide many more new extragalactic counterparts.

This research has made extensive use of the SIMBAD database, operated at CDS, Strasbourg (France), of the HEASARC service, provided by NASA/GSFC, and of the NED database operated by the JPL-NASA, Caltech. This work has been supported by NASA contract NAS5 -29298 to UCB.

\section{REFERENCES}

Bowyer, S., Lieu, R., Lampton, M., Lewis, J., Wu, X., Drake, J. J., \& Malina, R. F. 1994, ApJS, 93, 569

BRUHWELLER, C., ET AL. 1995, in prereparation

CZerny, B., \& ŻICKY, P. T. 1994, ApJ, 431, L5

DiCKEY, J. M., \& LoCKMAN, F. J. 1990, ARA\&A, 28, 215

Elvis, M., Lockman, F. J., \& WIIKEs, B. J. 1989, AJ, 97, 777

Fruscione, A., BowYer, S., KöNigl, A., \& KAHN, S. M. 1994, 422, L55

Fruscione, A. 1995, ApJ, submitted

KaAstra, J. S., Roos, N., MEwe, R. 1994, A\&A, in press

Königl, A., KartJe, J. F., Bowyer, S., KahN, S. M., \& Hwang, C. -Y. 1995, ApJ, in press

MalinA, R. F. ET AL. 1994, AJ, 107, 751

Marshall, H. L., Carone, T. E., \& Fruscione, A. 1993, ApJ, 414, L53

Marshall, H. L., Fruscione, A., \& Carone, T. E. 1995, ApJ, 439, 90 MFC

Marshall, H. L., Carone, T. E., Shull, J. M., Malkan, M. A., Elvis, M. 1995b, ApJ, submitted

PoUnds, K. A. ET AL. 1993, MNRAS, 260, 77

Stark, A. A., Gammie, C. F., Wilson, R. W., Bally, J., Linke, R. A., Heiles, C., \& HuRwiTZ, M. 1992, ApJS, 79, 77

Vennes, S., Polomski, E., Bowyer, S., \& Thorstensen, J. R. 1995, ApJ, in press

WALTER, R., \& FINK, H. H. 1993 A\&A, 274, 105

WIKEs, B. J., \& ElVis, M. 1987 ApJ, 323, 243 